УДК 338.984

http://doi.org/10.36906/KSP-2020/24

Гайнуллина Э.Р., Бурганов Р.А.

Казанский государственный энергетический университет

2. Казань, Россия

\title{
КОНКУРЕНТНАЯ СТРАТЕГИЯ ФИРМЫ В ИЗМЕНЯЮЩИХСЯ УСЛОВИЯХ СРЕДЫ
}

Аннотация. Рассматриваются вопросы формирования конкурентной стратегии фирмы, создания условий для обеспечения конкурентоспособности в изменяющихся условиях среды. Проведенное исследование позволяет сделать выводы, что для достижения эффективного долгосрочного функционирования и развития фирмы необходимо уделять внимание анализу вешней среды.

Ключевые слова: конкуренция; конкурентная стратегия; стратегическое планирование.

Бизнес-планирование является одним из основных направлений стратегического планирования. Все компании, находящиеся в высококонкурентной и быстро меняющейся ситуации, должны не только ориентироваться на внутреннее состояние дел в компании, но и формировать долгосрочную стратегию выживания, позволяющую им идти в ногу с изменениями, происходящими в их среде.

Адаптация к конкурентным условиям становится одной из главных задач менеджмента компании. Она проходит в рамках системного комплексного анализа деятельности конкурентов, разработки стратегии конкурентного поведения на рынке и реализации мероприятий, предусмотренных бизнес-планом предприятия. Главное здесь - обеспечить преимущества перед конкурентами.

Конкурентные стратегии являются важной и неотъемлемой частью современных рыночных отношений. Компания или организация строит свою конкурентную стратегию исходя из своего положения на рынке, т. е. является ли она лидером рынка, кандидатом на лидерство, последователем или обитателем ниши. Она анализирует своих конкурентов и оценивает свои возможности, и только после этого выбирает для себя подходящую конкурентную стратегию. Деловая практика показала, что не существует единой для всех компаний конкурентной стратегии. В то же время существует ряд основополагающих принципов адаптации системы управления предприятием к конкурентной среде [1].

Каждый сегмент характеризуется оригинальным потребительским поведением, а это значит, что у него есть своя конкурентная стратегия, которую компания может наилучшим образом реализовать в данном сегменте. В соревновании вы можете придерживаться наступательных и оборонительных стратегий. Любое конкурентное преимущество постоянно подвергается нападкам со стороны конкурентов, особенно богатых ресурсами. Есть две основные причины сходиться «врукопашную» с конкурентами, противопоставляя друг другу конкурентные преимущества. Первая - это попытка завоевать рыночное 
пространство, превзойдя сильные стороны более слабого конкурента. Атака более слабого соперника в момент его максимальной силы приносит уверенную победу и главное положение в соревновании. Другая причина - необходимость свести на нет конкурентные преимущества одного или нескольких конкурентов. Критерием успеха этой тактики является сравнение стоимости атаки с полученными выгодами. Атакуя слабые стороны соперника, атакующий переориентирует свои сильные стороны и ресурсы непосредственно на слабые стороны противника. Атаки на слабые стороны конкурентов имеют больше шансов на успех, чем атаки на их сильные стороны. На рынке все фирмы могут стать объектами атак со стороны конкурентов (включая новых участников рынка и фирмы, стремящиеся улучшить свои позиции).

Конкурентная стратегия компании должна включать в себя подходы к бизнесу и инициативы, которые она использует для привлечения клиентов, конкуренции и укрепления своих позиций на рынке.

Любая стратегия базируется на конкурентных преимуществах, которые позволяют компании иметь маржу прибыли выше средней для данной отрасли или сегмента рынка и завоевывать прочные позиции на рынке. Поэтому именно стратегии важны в этом отношении - достижение конкурентных преимуществ или конкурентные стратегии.

Конкурентная стратегия - это стремление компании занять конкурентную рыночную позицию в отрасли, т. е. на главной арене, где борются конкуренты. Конкурентная стратегия направлена на достижение стабильного и прибыльного положения, позволяющего компании противостоять давлению тех сил, которые определяют конкуренцию в отрасли, путем усиления ее конкурентных преимуществ перед конкурентами на рынке [2].

Как выбрать стратегию? Рентабельность отрасли является одним из важных факторов, определяющих выбор конкурентной стратегии. Второй основной задачей при выборе конкурентной стратегии является позиционирование компании в рамках конкретной отрасли. В зависимости от позиционирования по отношению к другим участникам рынка, ее доходы будут выше или ниже, чем в среднем по отрасли. Компания, занявшая выгодную позицию, получит высокую прибыль, даже если структура отрасли будет неблагоприятной, а средние показатели рентабельности из-за этого обстоятельства будут низкими.

Основой долгосрочного успеха компании является сильное конкурентное преимущество. Несмотря на то, что каждая компания имеет большое количество сильных и слабых сторон по сравнению со своими конкурентами, им предоставляется возможность иметь, как правило, только два вида конкурентных преимуществ: низкие издержки и дифференциацию продукции. Два основных типа конкурентных преимуществ в сочетании с областью, в которой компания пытается достичь этих преимуществ, позволяют ей разработать три наиболее распространенных конкурентных стратегии, которые могут быть использованы для достижения уровня эффективности, превышающего средний по отрасли: лидерство в минимизации затрат, дифференциация и фокусировка. Существует два типа политики фокусирования: фокусирование на затратах и фокусирование на дифференциации.

Главное, что нужно понимать о самых общих стратегиях, - это то, что каждая из этих стратегий по существу ориентирована на получение определенных конкурентных преимуществ, и для того, чтобы достичь этих преимуществ, компания должна сделать выбор, т. е. решить, какой тип конкурентных преимуществ ей нужен и в каком масштабе компания будет достигать этих преимуществ. Быть «всем для всех» невозможно - это стратегический 
рецепт для посредственной и неэффективной деятельности; это часто означает, что компания не имеет никаких конкурентных преимуществ.

Из трех наиболее распространенных стратегий минимизация затрат, пожалуй, самая очевидная и понятная. В рамках этой стратегии компания ставит своей целью наладить достаточно недорогое производство товаров в отрасли. Обычно такая компания имеет широкое поле деятельности: компания обслуживает несколько сегментов отрасли, по возможности захватывая смежные отрасли [3]. Зачастую только такая широкая сфера деятельности позволяет компании добиться лидерства в минимизации затрат. Источники стоимостных преимуществ могут быть очень разнообразными, и они варьируются в зависимости от типа отрасли. Это может включать в себя повышение производительности за счет экономии, за счет эффекта масштаба, патентованные технологии, особые права доступа к источникам сырья и некоторые другие факторы. Если компании удалось добиться неоспоримого лидерства в снижении потерь и сохранить это преимущество в течение длительного времени, то показатели такой компании значительно превысят среднерыночный уровень, но при условии, что компания сможет удерживать цены на свою продукцию на среднем для отрасли уровне или на уровне чуть выше его. Компания-лидер по снижению издержек благодаря этому преимуществу будет получать высокую прибыль даже по ценам, сопоставимым с ценами конкурентов, или по более низким ценам, чем у конкурентов. Хотя такая фирма не должна забывать об основах дифференциации. Продукт компании должен оцениваться потребителями как сопоставимый с продукцией конкурентов или хотя бы вполне приемлемый, иначе компания, даже будучи лидером по минимизации издержек, будет вынуждена существенно снижать цены на товары, чтобы продажи достигли достаточных показателей. И это может свести на нет все преимущества, получаемые от благоприятной позиции сокращения расходов.

Поэтому, сколько бы фирма ни ожидала конкурентных достижений в виде снижения издержек, она все равно должна достичь равенства или хотя бы приблизительного равенства в основах дифференциации своей продукции по отношению к продукции конкурентов только в этом случае фирма может достичь показателей эффективности, превышающих среднерыночный уровень. Равенство в основах дифференциации позволяет ведущей фирме в минимизации издержек естественным образом трансформировать свое преимущество в низких издержках в высокую прибыль - причем выше, чем у ее конкурентов. Но даже если базы дифференциации примерно равны, низкие цены, необходимые для получения контроля над необходимой долей рынка, не влияют на преимущества лидера в минимизации издержек, это означает, что лидер получает более высокие доходы, чем в среднем по рынку.

Регулярность стратегии лидерства в минимизации издержек обычно требует, чтобы фирма стала единоличным лидером, а не просто частью группы тех, кто стремится захватить эту позицию. Некоторые компании, отказавшиеся признать этот факт, допустили серьезную стратегическую ошибку. Когда есть несколько претендентов на позицию лидера в минимизации издержек, конкуренция между ними становится особенно жесткой, ведь каждый, даже самый маленький, фрагмент рынка начинает иметь решающее значение. И пока одна из организаций не возьмет на себя инициативу и не «уговорит» других конкурентов изменить свою стратегию, последствия этой борьбы за прибыльность (а также за структуру отрасли в долгосрочной перспективе) могут стать очень вредными, и это было в случае с несколькими нефтехимическими компаниями. Поэтому стратегия лидерства в 
минимизации издержек в основном базируется на приоритетном праве иметь некоторое превосходство, и компания вынуждена отказаться от этого права, если только в какой-то момент она не сможет полностью изменить свое положение в плане издержек за счет крупных научно-технических достижений.

Второй из общих конкурентных стратегий является стратегия дифференциации, которая заключается в том, что компания пытается захватить уникальное положение в определенной отрасли, придавая продукту характеристики, которые будут оценены большим количеством потребителей. Там может быть один или несколько таких данных или атрибутов - главное, чтобы они действительно были важны для клиентов.

В этом случае организация, продукция которой удовлетворяет определенные потребительские потребности благодаря этим атрибутам, позиционирует себя определенным уникальным образом, и наградой за эту уникальность является готовность потребителей платить высокие цены за продукцию компании.

Дифференциация может быть основана на уникальных свойствах самого продукта, особенностях реализации, специальных маркетинговых подходах, а также на множестве других факторов.

Компания, которая способна дифференцировать продукцию определенным образом и поддерживать выбранное направление в течение длительного периода времени, будет работать более эффективно, чем среднестатистическая компания в этой отрасли, но только в том случае, если маржа на продукцию компании превысит дополнительные затраты на дифференциацию, т. е. сделает продукт уникальным. Поэтому компания, которая выбирает стратегию дифференциации, должна постоянно искать новые способы дифференциации способы, которые могут генерировать прибыль, превышающую стоимость самой дифференциации. Но компания, идущая по пути дифференциации, не должна забывать и о затратах: любая, даже самая высокая маржа ни к чему не приведет, если компания займет позицию, не выгодную с точки зрения затрат. Поэтому, если фирма выбирает в качестве своей стратегии дифференциацию, она должна стремиться к равенству или приблизительному равенству издержек по отношению к своим конкурентам, сокращая издержки во всех областях, которые непосредственно не связаны с выбранным направлением дифференциации.

Логика стратегии дифференциации требует, чтобы компания основывала дифференциацию на атрибутах продукта, которые отличают ее от продукта конкурирующих компаний. Если компания хочет платить высокую цену за свою продукцию, она должна быть действительно уникальной или восприниматься клиентами как уникальная. Однако, в отличие от стратегии лидерства по затратам, реализация стратегии дифференциации не требует наличия только одного лидера в группе. В этом случае может существовать несколько компаний, которые успешно реализуют стратегию дифференциации, при условии, что продукция в этой отрасли имеет несколько параметров, которые особенно ценятся потребителями.

Третья общая стратегия конкуренции-- это стратегия фокуса. Эта стратегия отличается от других: она основана на выборе узкой области конкуренции внутри конкретной отрасли. Компания, избравшая стратегию фокусирования, выбирает определенный сегмент или группу отраслевых сегментов и направляет свою деятельность на обслуживание только этого сегмента или сегментов. Оптимизируя свою стратегию в соответствии с целевыми 
сегментами, компания пытается получить определенные конкурентные преимущества в этих сегментах, хотя она может не иметь общих конкурентных преимуществ во всей отрасли.

Существует два типа стратегий фокусирования. Ориентация на издержки - это стратегия, при которой компания, работая в своем целевом сегменте, пытается получить преимущество за счет низких издержек. Фокусируясь на дифференциации, компания дифференцируется в своем целевом сегменте. Оба варианта стратегии основаны на характеристиках, отличающих выбранный целевой сегмент от других сегментов отрасли. Целевой сегмент, скорее всего, объединяет как клиентов с особыми потребностями, так и системы производства и продаж, которые наилучшим образом отвечают им и отличаются от отраслевых стандартов на этой основе. Ориентируясь на издержки, компания пользуется различиями в их структуре в разных отраслях промышленности, а ориентируясь на дифференциацию, компания извлекает выгоду из того, что в определенных сегментах рынка существуют особые группы покупателей с особыми потребностями. Наличие таких различий в структуре затрат и потребительском спросе говорит о том, что эти сегменты слабо обслуживаются конкурентами с широкой специализацией - такие компании обслуживают эти особые сегменты наравне со всеми остальными. В этом случае компания, избравшая стратегию фокуса, получает конкурентные преимущества, полностью сосредоточив свою работу на данном сегменте. Неважно, узкий это сегмент или широкий: суть стратегии фокусирования заключается в том, что компания генерирует доход за счет особенностей этого сегмента, отличающих ее от других секторов отрасли. Узкой специализации самой по себе недостаточно для достижения компанией показателей эффективности, которые будут выше среднерыночных.

Компания, выбравшая фокус в качестве конкурентной стратегии, имеет существенное преимущество перед конкурентами с широкой специализацией, а именно: такая компания может выбрать направление оптимизации - дифференциацию или снижение издержек. Например, вполне возможно, что конкуренты не обслуживают определенный сегмент рынка достаточно хорошо, чтобы удовлетворить потребности клиентов в этом секторе, и тогда у компании есть отличные возможности сосредоточиться на дифференциации. С другой стороны, конкуренты с широкой специализацией, скорее всего, потратят слишком много денег и усилий на обслуживание этого сегмента, а значит, их затраты на удовлетворение потребностей клиентов в этом сегменте слишком высоки. В этом случае у компании есть возможность выбрать ориентацию на издержки, ведь можно снизить издержки, потратив деньги исключительно на удовлетворение потребностей клиентов в данном сегменте, и не более того.

Если выбранный компанией целевой сегмент не отличается от других сегментов, стратегия фокусирования не даст необходимых результатов. Показатели эффективности компании, выбравшей стратегию фокусирования, будут выше среднеотраслевых, если:

а) компания способна достичь стабильного лидерства в минимизации издержек в своем сегменте (фокусирование на издержках) или дифференцировать свой продукт в этом сегменте (фокусирование на дифференциации);

б) для этого сегмента он будет заманчивым с точки зрения его структуры. Структурная привлекательность сегмента является необходимым условием, так как некоторые сегменты в отрасли будут заведомо менее прибыльными, чем другие. 


\section{КУЛЬТУРА. НАУКА. ОБРАЗОВАНИЕ}

Каждая из наиболее распространенных стратегий конкуренции - это полностью независимый подход к получению конкурентных преимуществ и способам их сохранения в течение длительного периода времени. Каждая стратегия сочетает в себе определенный тип конкурентного преимущества, которого компания пытается достичь, а также масштаб стратегической цели [4].

Выбор конкурентной стратегии предприятия относится к этапу определения альтернатив и выбора основной стратегии предприятия, в рамках которой организация будет развиваться и осуществлять свою деятельность.

Таким образом, для успешного функционирования предприятия в отрасли необходимо своевременно и оперативно реагировать на изменяющиеся условия внешней среды и выбирать конкурентную стратегию для достижения конкурентного преимущества перед другими предприятиями отрасли.

\section{Литература}

1. Агапова М.А. Управление конкурентными преимуществами предприятия // Карельский научный журнал. 2017. № 3 (20). С. 81-84.

2. Григорчук А.В. Методы оценки конкурентной позиции и конкурентных преимуществ предприятий // Научно-практические исследования. 2017. № 8 (8). С. 27-31.

3. Носова Н. С. Конкурентная стратегия компании, или Маркетинговые методы конкурентной борьбы. М., 2013. 256 с.

4. Рохчин В.Е., Ветрова Е.Н. Управление стратегической конкурентоспособностью промышленного предприятия на основе развития его потенциала / Под ред. А.Е. Карлика. СПб., 2018. 262 с.

(СГайнуллина Э.Р., Бурганов Р.А., 2020 